\title{
Crisis social y política en Chile: la demanda por acceso y cobertura universal de salud
}

\author{
Osvaldo Artaza Barrios ${ }^{1}$ y Claudio A. Méndez ${ }^{2}$
}

Forma de citar Artaza Barrios O, Méndez CA. Crisis social y política en Chile: la demanda por acceso y cobertura universal de salud. Rev Panam Salud Publica. 2020;44:e16. https://doi.org/10.26633/RPSP.2020.16

RESUMEN

En Chile se ha producido un desarrollo económico que no ha resuelto profundas desigualdades. Hoy en día, se intenta resolver una intensa crisis política y social que irrumpe de modo explosivo en octubre de 2019 motivada por un alza del transporte público. Una década después de que se iniciara la implementación de la última reforma al sistema de salud, este sigue siendo un ámbito prioritario en la preocupación de la población y parte de las actuales demandas ciudadanas. Desde el movimiento social, se proponen nuevas reformas que requerirán de consensos para ser viables desde un punto de vista técnico y político. Los cambios necesarios en el ámbito de la salud son impostergables. Entre otras medidas, es preciso implementar transformaciones en el sector prestador, tanto público como privado, en la lógica de un modelo estructurado en redes integradas y basado en la estrategia de la atención primaria de salud. Esto permitirá la consecuente alineación de los recursos humanos y financieros con el acceso y cobertura de salud universal y, de esta manera, resolver de manera efectiva y eficiente las necesidades de salud de las personas y sus comunidades.

Palabras clave Sistemas de salud; modelo de atención; acciones integradas de salud; atención primaria de salud; Chile.

Chile es un país situado en el Cono Sur de América Latina, tiene 18 millones de habitantes, un índice de desarrollo humano alto $(0,82), 8 \%$ de la población en situación de pobreza, expectativa de vida de 79,9 años y una tasa de mortalidad infantil de 7 por cada mil nacidos vivos. Sin embargo, aún es un país inequitativo (el índice de distribución del ingreso de Gini es de 46,6\%), con un gasto público en salud insuficiente $(4,2 \%$ del producto interno bruto) y un gasto de bolsillo elevado (33\%) (1).

Hoy en día, existe en Chile un sistema segmentado e inequitativo de salud (2), en el que conviven un fondo público que da cobertura a $80 \%$ de la población (Fondo Nacional de Salud, FONASA) con múltiples seguros privados (instituciones privadas de salud previsional, ISAPRES) para 18\% de la población (3). En el ámbito hospitalario, el sector prestador es, en su mayoría público, mientras que, en la atención ambulatoria, lo es por partes iguales (público y privado). El sector privado sigue una lógica de mercado e incentiva el consumo de prestaciones. Por su parte, el sector público presenta dificultades para resolver las necesidades de personas y sus comunidades debido, entre otros problemas, a un financiamiento insuficiente, fallas en su organización y la presencia de un fenómeno de captura múltiple: presiones por parte del mercado de la salud con el fin de generar más consumo de prestaciones, por parte de afanes clientelares de partidos políticos, por la presencia de intereses corporativos fuertes y por la hegemonía de un paradigma biomédico hospitalario céntrico (4).

Se revisan en forma somera los procesos de reforma de las últimas décadas y en perspectiva de la profunda crisis social actual en Chile, y se plantean los principales desafíos para resolver las necesidades en salud de personas y sus comunidades.

\section{LAS REFORMAS AL SISTEMA DE SALUD CHILENO Y SUS DESAFÍOS}

De manera tradicional, Chile ha contado con un sistema de salud bien organizado y gobernado en forma efectiva (5), que

\footnotetext{
1 Facultad de Ciencias de la Salud, Universidad de las Américas, Chile. $\triangle$ Osvaldo Artaza Barrios, oartaza@udla.cl
} 
ha estado sujeto a tres procesos de reforma. El primero (1952) dio origen al Sistema Nacional de Salud (SNS). El segundo tuvo lugar durante la dictadura militar (1980), en ese período se fragmentó el SNS y la atención hospitalaria quedó en manos de 29 servicios descentralizados (que dan cobertura al $80 \%$ de la población) con un total de 2,2 camas por mil y entregó la atención de primer nivel (conformado por 2179 centros de atención) a la administración municipal (345 en el país). Además, creó un fondo público de salud (Fondo Nacional de Salud, FONASA) e impulsó el desarrollo del sector privado con la creación de seguros privados de salud (instituciones privadas de salud previsional, ISAPRES).

En el tercer y último proceso de reforma (2002-2005), el objetivo era avanzar en temas de equidad, materialización de derechos, empoderamiento de los usuarios e integración de políticas sociales. A tal fin, se promulgó un conjunto de garantías exigibles en salud en oportunidad, acceso, protección financiera y calidad, conocido como el plan de Acceso Universal con Garantías Explícitas (AUGE). También se aumentó la capacidad regulatoria del Estado sobre los actores para asegurar el cumplimiento de las garantías. Esto se logró mediante la transformación de la Superintendencia de ISAPRES, que se había creado a principios de los años noventa para regular a las aseguradoras privadas, en una nueva entidad (Superintendencia de Salud) para fiscalizar el cumplimiento de los derechos (las garantías) en el sector público como privado a nivel de seguros (intendencia de seguros) y para garantizar la calidad de prestadores individuales e institucionales (intendencia de prestadores) $(1,6,7)$.

El sector salud se ha constituido en uno de los elementos esenciales del estallido social en Chile iniciado el año 2019 (8), lo que es una clara señal de expectativas no resueltas. La última reforma ha tenido efectos positivos, como instalar el concepto de derechos en salud, así como avances en equidad y en resultados sanitarios en algunas áreas específicas (1). Pero también existen fundadas críticas de diversos sectores y el claro descontento de la población, relacionado sobre todo con la persistencia de inequidades en salud, las dificultades para acceder a medicamentos y la atención en problemas de salud no priorizados en el plan de garantías explícitas y en la no resolución de aspectos pendientes en el aseguramiento privado, entre otros (1).

En el momento político actual se observa una presión enorme para una agenda de reformas políticas y sociales muy amplias en el ámbito de las pensiones, la educación, los salarios y las condiciones laborales, en paralelo al desarrollo de un proceso para generar una nueva constitución política. Esto puede dejar un margen escaso para orquestar cambios relevantes en el ámbito de la salud, con el riesgo de que estos se acoten a mejoras declarativas o limitadas al ámbito del aseguramiento, y pueden quedar afuera, entre otras, transformaciones en la prestación de servicios y su modelo de atención. Esto obliga a generar mayores espacios para desarrollar evidencia y consensos sociales y políticos sobre las mejoras integrales que requiere el sistema de salud chileno.

Los cambios propuestos por agrupaciones de la sociedad civil en el marco del movimiento social (9) que se desarrolla en Chile se pueden resumir en modificaciones constitucionales que aseguren el derecho a la salud, alcanzar un gasto público de al menos $6 \%$ del producto, aumentar financiamiento al primer nivel de atención y mancomunar fondos a partir del actual Fondo Nacional de Salud para generar un "seguro único de salud" (10), en el que las ISAPRES sean solo seguros complementarios.

El proceso que se abre para una nueva constitución, a partir de la presión social, será complejo y obligará a generar consensos sobre la comprensión que la sociedad chilena tiene sobre el concepto de "derecho a la salud", dada la necesidad de reunir dos tercios de los congresales para aprobar cualquier materia de orden constitucional. Dado que es imposible garantizar el estar sano, ni adecuado reducirlo al campo de las enfermedades y al de los meros servicios de atención médicos, en la labor constituyente, el derecho a la salud deberá ser necesariamente comprendido en un espacio amplio, que guarde relación con la integralidad de los requerimientos socioeconómicos que determinan la posibilidad de que todas las personas -plenas en dignidad- puedan desarrollar todo su potencial y gozar el máximo bienestar en las múltiples dimensiones de lo humano. Desde dicha construcción, las instituciones del Estado tendrán que salir fortalecidas en su capacidad de regular el mercado y favorecer condiciones más saludables de vida para que, junto a las personas, las familias y sus comunidades, se pueda actuar de modo más efectivo sobre los determinantes sociales de la salud. Cualquier definición reduccionista arriesga a ser solo declarativa o a dejar en manos de los jueces su interpretación práctica.

Con respecto a la exigencia de un aumento necesario del gasto público en salud, esencial para disminuir el alto componente de gasto de bolsillo (sobre el 30\%) (11), se deberá también mejorar la calidad y eficiencia (12) de dicho gasto. Más recursos, requeridos con urgencia, no serán suficientes si no se aborda en Chile una transformación profunda a los prestadores, su modelo de atención, de gestión, los mecanismos de pago y las políticas de desarrollo de capital humano para el sector prestador público y privado. Es evidente que hay un gran desafío pendiente para que el sistema de salud chileno gane en calidad del gasto, con una estructuración efectiva en redes integradas basadas en la estrategia de la atención primaria a la salud (APS) (13).

La constitución de un seguro único público, que es una de las propuestas de los grupos sociales, conlleva en Chile innumerables desafíos técnicos, especialmente en el Fondo Nacional de Salud, tales como la definición de un plan de salud universal, cambios en su gobernanza actual que permita generar confianza en todos los sectores ciudadanos, y modificaciones en su estructura y competencias para articular en forma adecuada a los prestadores públicos y privados y asegurar la factibilidad del plan universal. Reconocer y aceptar la pluralidad de prestadores que existe en Chile y diseñar un modo racional de incorporarlos de modo aprovechar su capacidad de oferta con sentido sanitario será fundamental para la viabilidad de la mancomunación de fondos.

Hay varios aspectos clave del sistema de salud chileno, que desafortunadamente han estado fuera de las demandas luego del estallido social. Este aún no completa una necesaria transición en la comprensión de las personas como sujetos de derechos, que no solo tienen una autonomía que se debe respetar, sino que son seres sociales que se vinculan en un territorio y que tienen un papel protagónico, junto a diversos actores y al apoyo de los servicios de salud, para la construcción de contextos para una vida buena y saludable, más allá de una participación meramente formal, utilitarista y clientelar (14). Junto a lo anterior, los servicios de atención en Chile, a pesar de 
los esfuerzos de muchos, siguen actuando desde un paradigma esencialmente curativo y hospitalo-céntrico y tratando a las personas ya sea como clientes en un mercado de prestaciones o como sujetos pasivos de asistencialismo (15), desde modelos verticales centrados en la enfermedad y con una mirada fragmentada de las necesidades humanas, separando "órganos enfermos, patologías" de las demás dimensiones comprendidas en el logro del mayor nivel de calidad de vida posible.

Más allá de las actuales demandas que se han manifestado en el llamado "estallido social", los desafíos son gigantescos para un proceso de transformación que está, hoy en día, muy en sus inicios. Del concepto de "órgano enfermo" y servicios fragmentados y capturados por visiones distintas a la de las necesidades de las personas y sus comunidades, debemos pasar a redes de colaboración entre muchos actores que se organizan, estructuran y se rediseñan de manera constante. Así se podrá brindar una respuesta integral a dichas necesidades, con la participación clave y central de las propias personas y sus comunidades.

Chile requiere de un modelo de atención común para todos los prestadores públicos o privados, que supere la relación de mercado por una de cooperación, que evite que las personas acudan de forma directa a un especialista o que el primer lugar de consulta sean los servicios de urgencias de los hospitales de agudos. El modelo de atención debe asegurar que toda persona acceda a un equipo de familia interdisciplinario, cercano, resolutivo, confiable y de alta calidad, centrado en las personas, las familias y sus comunidades con mirada de territorio. Para ello, hay que desarrollar nuevas soluciones y dispositivos ambulatorios en el propio hábitat de las personas, potenciar la creación de establecimientos de alta complejidad ambulatorios, de internación comunitaria para largas estadías y de manejo en el domicilio de personas. Es necesario desarrollar redes locales eficaces de cuidados sanitarios y sociales, para el manejo de requerimientos de adultos mayores, salud mental, rehabilitación y de cuidados paliativos y de largo plazo; incorporar nuevos elementos de gestión y gobernanza en las redes para la integración efectiva de los actores y asegurar la labor intersectorial y la continuidad del cuidado, y aumentar de manera sustantiva la inversión en el primer nivel de atención mucho más allá del actual 23\% del total del gasto (16), de modo hacerlo más resolutivo. Sin duda, esto contribuirá a una mejor percepción de su calidad y a un mayor costo efectividad. Un cambio de modelo, además de modificaciones en estructura y gobernanza de las redes de prestación, implica cambios en la formación de los trabajadores de la salud hacia una comprensión centrada en las personas y las comunidades de su labor y de condiciones e incentivos correctos para contar con los mejores profesionales y técnicos como integrantes de equipos interdisciplinarios, junto a las tecnologías sanitarias probadamente costo efectivas, y el apoyo continuo de las especialidades al primer nivel de atención, de forma se pueda realizar prevención y evitar hospitalizaciones y requerimientos de manejo de especialidad cambiando un modelo cuya ineficacia y falta de respuesta están probadas (1).

Los múltiples desafíos en el sector prestador público giran sobre la conformación, gobernanza e integración de las redes $(17,18)$ y sobre los mecanismos de pago (19) (en particular al recurso humano), entre muchos tópicos. Con respecto a los hospitales públicos, la preocupación ha girado sobre su eficiencia en el uso de los recursos (20), ya que por una parte los aumentos constantes en su financiamiento están condenados a nunca alcanzar sus "costos reales" y a no traducirse en aumentos de su productividad, lo que se explica -además de los factores demográficos y epidemiológicos- por la tendencia permanente de los equipos clínicos de agregar (muchas veces por presiones de la industria) nuevas tecnologías más costosas, no siempre con estudios de costo efectividad que lo avalen, lo que se traduce en igual actividad pero a costos siempre crecientes. Dada la coexistencia en Chile de prestadores privados, los intentos por aumentar el desempeño de los hospitales públicos solo a través del aumento tanto del número de médicos como de su remuneración no ha tenido el resultado esperado. Una solución -no exenta de complejidad política- es revisar la forma de relación contractual con el cuerpo médico de manera que se relacione con resultados esperados y se pueda evitar estas distorsiones e incentivos incorrectos.

\section{CONCLUSIONES}

Las movilizaciones sociales iniciadas en Chile en octubre del año 2019, con profundas exigencias para poner fin a inequidades y abusos, constituyen un espacio de posibilidades con el fin de avanzar hacia el acceso y la cobertura universal a la salud.

Chile es un país con un fuerte determinismo social de la calidad de vida de las personas y una predominancia de las enfermedades crónicas no transmisibles. Los pendientes más relevantes son entre otros, construir un nuevo contrato social en torno a una comprensión común del derecho a la salud, mejorar las capacidades del estado chileno para actuar sobre las determinantes sociales de la salud, mancomunar fondos para mejorar la equidad en el financiamiento, aumentar el gasto público y su calidad y eficiencia, pasar de una participación social solo como mecanismo formal de control social, a una instancia que permita una real inclusión, autonomía y diálogo, y cambiar el paradigma de los prestadores que siguen organizados con base en "problemas y patologías", hacia uno efectivamente centrado en necesidades integrales de personas y sus comunidades.

El debate sobre los cambios necesarios debe colocar en el centro las necesidades de salud más urgentes de personas y comunidades y, con base en ellas, implementar el mejor modo de organizarnos para cumplir con dichos requerimientos. Escuchar el descontento ciudadano y poner en el centro las necesidades de las personas y sus comunidades nos obliga a aprender de los logros alcanzados y a preguntarnos sobre los próximos pasos. Lo importante es hacerlo con base en la evidencia, esforzándonos por dar gobernanza a un sector presionado por intereses de múltiples actores, lo que requiere no solo de consensos técnicos, sino también de amplios acuerdos sociales. Esto último parece ser la lección esencial del reciente estallido social en Chile.

Contribución de los autores. Todos los autores han participado de modo balanceado en todas las fases del artículo.

\section{Conflicto de intereses. Ninguno declarado por los autores.}

Declaración. Las opiniones expresadas en este manuscrito son responsabilidad del autor y no reflejan necesariamente los criterios ni la política de la RPSP/PAJPH y/o de la OPS. 


\section{REFERENCIAS}

1. Artaza O, Lastra J. La nueva reforma que necesita Chile: la reforma de los prestadores. Cuad MEd Soc (Chile). 2018;58(1):27-33.

2. Sánchez RH, Albala C. Desigualdades en salud: adulto en comunas del Gran Santiago. Rev Med (Chile). 2004;132(4):453-60. http://dx. doi.org/10.4067/S0034-98872004000400007

3. Crespo C. Chile: nuevos desafíos sanitarios e institucionales en un país en transición. Rev Panam Salud Publica. 2018;42:e137. https:/ / doi.org/10.26633/RPSP.2018.137

4. Urriola C, Infante A, Aguilera I, Ormeño H. La reforma de salud chilena a diez años de su implementación. Salud Publica de México. 2016;58(5):514-21. Disponible en: https:/ / www.redalyc.org/articulo. oa?id=10647486004

5. Organización para la Cooperación y el Desarrollo Económicos (OCDE). Estudios de la OCDE sobre salud pública: Chile. Santiago: OCDE y Ministerio de Salud de Chile; 2019. Disponible en: https:/ / www.oecd.org/health/health-systems/Revisi\%C3\%B3nOCDE-de-Salud-P\%C3\%BAblica-Chile-Evaluaci\%C3\%B3n-yrecomendaciones.pdf

6. Goic A. El Sistema de Salud de Chile: una tarea pendiente. Rev Med Chile. 2015;143(6):774-86. http://dx.doi.org/10.4067/ S0034-98872015000600011

7. Cid C, Uthoff A. La reforma a la salud pendiente en Chile: reflexiones en torno a una propuesta de transformación del sistema. Rev Panam Salud Publica. 2017;41:e170. https://doi.org/10.26633/ RPSP.2017.170

8. Paúl F. Protestas en Chile: las 6 grandes deudas sociales por las que muchos chilenos dicen sentirse "abusados". BBC News Mundo: 2019, octubre 21. Disponible en: https://www.bbc.com/mundo/ noticias-america-latina-50124583

9. Mesa Social por el Derecho a la salud (sitio web). Disponible en: http://derechosalud.cl/

10. Colegio Médico de Chile. Propuestas para mejorar la salud del país desde los colegios, gremios y usuarios del sector; 2019. Disponible en: http:/ / www.colegiomedico.cl/propuestas-para-mejorar-la-saluddel-pais-desde-los-colegios-gremios-y-usuarios-del-sector /

11. Organización para la Cooperación y el Desarrollo Económicos (OCDE). Health at a Glance 2015. Disponible en: http://www. oecd.org/chile/Health-at-a-Glance-2015-Key-Findings-CHILE-InSpanish.pdf

12. Artaza O. Editorial. Salud Pública de México. 2016;58(5):493-5. Disponible en: http://saludpublica.mx/index.php/spm/article/ view /8183
13. Organización Panamericana de la Salud (OPS). CD53/5. Línea estratégica 1: Ampliar el acceso equitativo a servicios de salud, integrales, de calidad, centrados en las personas y las comunidades. Documento $53^{\circ}$ Consejo Directivo; 2014.

14. Méndez CA, Vanegas López JJ. La participación social en salud: el desafío de Chile. Rev Panam Salud Publica. 2010;27(2):144-8.

15. Benavides P, Castro R, Jones I. Sistema público de salud. Situación actual y proyecciones fiscales 2013-2050". Dirección de Presupuestos de Chile (DIPRES); 2013. Disponible en: http:/ / bibliotecadigital. dipres.gob.cl/handle/11626/8665

16. Püschel K, Téllez A, Montero J, Brunner A, Peñaloza B, Rojas MP, et al. Hacia un nuevo modelo de atención primaria en salud. Centro de Estudios Públicos. 2013;130:23-52. Disponible en: https:/ /www.cepchile.cl/cep/estudios-publicos/n-121-a-la-150/ estudios-publicos-n-130-2013/hacia-un-nuevo-modelo-deatencion-primaria-en-salud-evaluacion-del

17. Centro de Estudios Públicos y Escuela de Salud Pública Universidad de Chile. Propuesta de modernización y fortalecimiento de los prestadores estatales de servicios de salud. Santiago de Chile: CEP; 2017 Disponible en: https://www.cepchile.cl/cep/site/artic/20170706/ asocfile/20170706104922/cep_udechile_propuesta_salud.pdf

18. Artaza-Barrios O, Toro-Devia O, Fuentes-García A, Alarcón-Hein A, Arteaga-Herrera O. Gobierno de redes asistenciales: evaluación de los Consejos Integradores de la Red Asistencial (CIRA) en el contexto de la reforma del sector salud en Chile. Salud Publica Mex. 2013;55(6):650-8. Disponible en: http://www.scielo.org.mx/scielo. php?script=sci_arttext\&pid=S0036-36342013001000014\&lng $=e s$

19. Vergara M, Martínez MS. Financiamiento del sistema de salud chileno. Salud Publica Mex. 2006;48(6):512-21.

20. Santelices C Emilio, Ormeño C Héctor, Delgado S Magdalena, Lui M Christopher, Valdés V Raúl, Durán C Lorena. Análisis de los determinantes de la eficiencia hospitalaria: el caso de Chile. Rev Med Chile. 2013;141(4):457-63. http://dx.doi.org/10.4067/ S0034-98872013000400006

Manuscrito recibido el 12 de noviembre de 2019. Aceptado para su publicación, tras revisión, el 27 de diciembre de 2019.

\section{Social and political crisis in Chile: the demand for universal access to health and universal health coverage}

ABSTRACT In Chile, economic development has not resolved deep inequalities. Today, attempts are being made to solve an intense political and social crisis sparked in October 2019 by a hike in public transport fares. A decade after initial implementation of the last health system reform, this continues to be a priority area of public concernand part of what citizens are now demanding. This social movement is proposing new reforms that will require consensus in order to be viable from a technical and political standpoint. The necessary changes in the health field cannot be postponed. Among other measures, it is necessary to implement transformations in health providers in both the public and private sectors, toward a model that is structured around integrated networks and based on a primary health care strategy. This will make it possible to align human and financial resources with universal access to health and universal health coverage, effectively and efficiently meeting the health needs of people and communities.

Keywords Health systems; health care models; intersectoral collaboration; primary health care; Chile. 


\section{Crise política e social no Chile: demanda por acesso universal à saúde e cobertura universal de saúde}

RESUMO O crescimento econômico alcançado no Chile não foi capaz de resolver as profundas desigualdades. O país tenta hoje encontrar uma saída à enorme crise política e social que irrompeu com violência em outubro de 2019 contra o aumento no preço da passagem do transporte público. Passada uma década desde a última reforma do sistema de saúde, a saúde continua sendo um tema prioritário que preocupa a população e figura entre as demandas atuais dos cidadãos chilenos. As novas reformas propostas pelo movimento social exigem o consenso técnico e político para serem viabilizadas. As mudanças necessárias em saúde são impreteríveis. Entre outras medidas, é preciso transformar o setor de prestação de serviços, tanto público como privado, criando um modelo estruturado em redes integradas baseado na estratégia de atenção primária à saúde. Isto possibilitará o consequente alinhamento dos recursos humanos e financeiros com o acesso universal à saúde e cobertura de saúde universal e, assim, atender com efeito e eficiência às necessidades de saúde das pessoas e suas comunidades.

Palavras-chave Sistemas de saúde; modelos de assistência à saúde; colaboração intersetorial; atenção primária à saúde; Chile. 